\title{
SOME CHALLENGES FACING NEUROREHABILITATION IN NIGERIA: STANDPOINT OF A NEUROPHYSIOTHERAPIST
}

\author{
Talhatu K Hamzat, Department of Physiotherapy, College of Medicine (UCH), \\ University of Ibadan, Nigeria
}

*Corresponding author: Talhatu K Hamzat, Honourary Consultant in Clinical Neurophysiotherapy, Department of Physiotherapy, College of Medicine (UCH), University of Ibadan PMB 5017 GPO Dugbe, 2000005, Ibadan Nigeria, Tel: +234-7031096849; E-mail: tkhamzat@comui.edu.ng

Received: April 26, 2016; Accepted: August 30, 2016; Published: August 31, 2016

\section{NEUROREHABILITATION}

Neurorehabilitation is the process of ensuring that any person presenting with neurological deficit lives a meaningful, qualitative and prolonged life while restoring function as much as possible (Owolabi and Hamzat, 2011). It involves multidisciplinary and multidimensional therapies which entails process of assessment, treatment planning, goal setting, therapeutic intervention and evaluation of outcome. This team effort becomes fruitful with contributions from specialties of neuromedicine/neurosurgery, occupational therapy, (Hamzat, 2013) ergotherapy, speech and language therapy, psychotherapy, visual rehabilitation, audiological rehabilitation, sexual therapy, relaxation and music therapy (Owolabi and Hamzat, 2011). Many developing countries like Nigeria, do not have the benefits of professionals in many of the listed sub-specialties and as such patients needing comprehensive rehabilitation are unable to enjoy the services.

Several factors from the obvious to the not-so obvious ones contribute to depriving patients the benefit of care and increasing the burden of neurorehabilitation in Nigeria. This submission is however delimited to three challenges, that of shortage of manpower, poor government policy and inter-professional rivalry-all from the prism of a neurophysiotherapist.

\section{MANPOWER}

There are few There are few neuro-rehabilitation experts in Nigeria. From neurophysician, speech therapist to neurophysiotherapists, the expert-patient ratio is unfortunately high. Using neurophysiotherapy as an example, a specialty that is arguably core to neurorehabilitation, in a previous submission (Hamzat, 2014), I had noted that a major challenge which the subspecialty of neurophysiotherapy face in Nigeria is lack of adequate manpower. Whereas there are about 2,500 physiotherapists in the Nigeria, only about 60 Physiotherapists practicing in the country had one of postgraduate certificates, diploma, Masters or PhD degrees in Neurophysiotherapy, a pattern that was attributed in part to preference for "less stressful" specialties and limited opportunities for training in the field. The story is not significantly different in other sub-specialties. For instance while 12 universities offer entry-level physiotherapy degree training opportunities in Nigeria, only one university provides such opportunity for occupational therapy in a country of about 180 million people. As already highlighted by Owolabi, the problem of scarce human resources in neurorehabilitation is not limited to physiotherapy. He noted that there is an average of 1 neurologist to one million people in South Saharan Africa compared to 1 to 100,000 in high income countries. In Nigeria, there are less than 50 occupational therapists and a paltry 10 speech therapists. Out of about 2800 physiotherapists that had been 
trained in Nigeria, nearly $35 \%$ are working overseas. Of those working in Nigeria, It is unfortunate that an infinitesimally small promotion is constituted by neurophysiotherapists. Efforts are however being made to improve opportunities for training of more neurorehabilitation professionals by the appropriate authorities.

\section{UNREGULATED TRANSPORT SYSTEM}

There appears to be a paradigm shift in pattern of disease from communicable to non-communicable diseases in Nigeria. Hypertension, diabetes mellitus, obesity and renal disease which are modifiable risk factors for stroke are reportedly on the rise. More significant however is the steady rise in prominence of road traffic accident-related neurological disorders as a burden on neurorehabilitation services. The apparently worsening mass transportation services, have resulted in a penchant for commercial motorcycles as a mode of mass transit in both urban and rural communities of Nigeria. The 2015 WHO Global Status Report on Road Safety showed that motorcyclists are particularly vulnerable and account for about $23 \%$ of all road traffic deaths. Sadly, there appears to be a lack of will on promulgation and/or enforcement of rules guiding operation of commercial motorcycle and promotion of safety on Nigeria roads. This "system-failure" has led to increased road-traffic related injuries and death on Nigerian roads. Those who survive the crashes commonly present with musculoskeletal injuries and many others are seen in our health facilities for management of life threatening/ modifying spinal cord and head injuries.

\section{TEAM SPIRIT}

A casual observation would reveal that in the few health facilities that parade a reasonably constituted neurorehabilitation team, intra-professional communication and relationship is far from adequate. It is common to note in many cases a master-subject relationship is the approach to relationship among the professionals, the "master" in virtually all instances is the physician/surgeon. The situation is such that opinions of other members of the team in planning and execution of plan of managing the patient are not considered by others. The idea of a cyclical relationship among all team members with the patient as the focus is alien. Interprofessional rivalry, denies patients the opportunity of full-blast expert services, as sometimes patient needing more physiotherapy in the expert opinion of the neurophysiotherapists are discharged from in-patient facility without any recourse to the carefully designed physiotherapy plan. It is also not uncommon for one member of the team to delve into the field of another team member, in total disregard for the fact that the person in whose field he is infringing is a specialist in his own right. This is often times is a result of lack of knowledge and understanding of the meaning, scope and peculiarities of the other team member's profession.

The working relationships, or lack of it, among health workers have been of interest to some researchers. Ogbimi and Adebamowo, who surveyed doctors and nurses working in four university teaching hospitals in Southern Nigeria had noted that the problem of poor inter-professional collaboration was posing a grave threat to the expected outcomes of team building in the healthcare sector. Their results showed that nursedoctor working relationships were significantly statistically affected by poor social interaction, staff shortages, activist unionism, disregard for ones profession as well as unsatisfactory hospital management and government policies. Their reported trend is unlikely to differ, were the survey to involve physiotherapists-and-doctors. There is an improving formal collaborative effort among neurorehabilitation professionals in Nigeria in the area of training and research. While physiotherapists and Occupational Therapists are co-trained in one of the Nigeria universities, other professionals such as neurologists, neurosurgeons and sociologists are involved in the training of neurophysiotherapists in many training centres in Nigeria. Likewise, neurophysiotherapists are involved in teaching of physicians specializing in neuromedicine in a faculty at the West African college of physicians. A cursory look at research publications in the field of neurorehabilitation in Nigeria would reveal some collaborative efforts, particularly between physiotherapists and physicians. These collaborative efforts often times enable better appreciations of what neurophysiotherapy have to offer neurologically ill patients. 
It also helps to build trust and better understanding among members of the team [World Health Organisation (2015)]. Such trust would enable members of the team to willingly share their knowledge and skills without fear of being ridiculed or not appreciated.

\section{CONCLUSION}

The field of neurorehabilitation is fairly a virgin one in Nigeria. The burden placed on this developing specialty by inadequate number of professionals, increasing number of road-traffic injury related neurological disorder and poor inter-professional relationship among neurorehabilitation team members in Nigeria was highlighted in this brief write-up.

\section{REFERENCES}

Hamzat, T.K. (2013). From Ward to Ward: The Neurophysiotherapist as a Returning Officer. An inaugural lecture delivered at the University of Ibadan, Nigeria. Ibadan, Ibadan University Press Publishing House. ISBN: 978-978-8456-20-9.

Owolabi, M.O., and Hamzat, T.K. (2010). Rehabilitation of the patient with neurological deficits. Archives of Ibadan Medicine 10(1-2): 65-70.

Ogbimi, R.I., and Adebamowo, C.A. (2006). Questionnaire survey of working relationships between nurses and doctors in university teaching hospitals in Southern Nigeria BMC Nursing. 5:2. doi: 10.1186/1472$6955-5-2$

Owolabi M.O. (2014). Changing Trend in need for Neurorehabilitation. In: Africa- A SCOTA analysis. How to Promote Neurorehabilitation in Africa- Going Forward. A paper presented at the 8th World Congress for Neurorehabilitation, 8 -13 April, 2014, Istanbul Turkey.

World Health Organisation (2015). Global Status Report on Road Safety 2015 WHO. Geneva, Switzerland. 